\title{
High Temperature Structure and Properties of Grain Boundaries - Insights Obtained from Atomic Level Simulations
}

\author{
P. KEBLINSKI
}

Materials Science and Engineering Department

Rensselaer Polytechnic Institute, Troy, NY, USA

\begin{abstract}
Molecular-dynamics simulations of grain boundaries in Si and fcc metals reveal that high-energy boundaries are disordered, even at low temperatures, with their local atomic structure very similar to that of bulk amorphous material. By contrast, low energy grain boundaries are crystalline all the way up to the melting point. Upon heating intergranular "confined amorphous" structures of high-energy grain boundaries exhibit reversible transition into universal, highly confined, liquid-like structure. High-temperature properties, such as the grain boundary diffusion therefore involve liquid-like mechanisms, with activation energies related to the diffusion process in the melt. By contrast to $\mathrm{Si}$ and fcc metals, high-energy diamond grain boundaries are more ordered structurally, but contain coordination disorder resulting from ability of carbon to change its hybridization from $s p^{3}$ to $s p^{2}$ character. Based on the insights obtained from our simulation of individual grain boundaries we were able to design nanocrystalline $3 \mathrm{D}$ microstructures, which allow to study grain boundary diffusion creep process by molecular-dynamics simulations. In order to prevent grain growth and thus to enable steady-state diffusion creep to be observed on a time scale accessible to molecular-dynamics simulations (of typically $10^{-9} \mathrm{~s}$ ), our input microstructures were tailored to (i) have a uniform grain shape and a uniform grain size of nm dimensions and (ii) contain only high-energy grain boundaries that, consistently with our studies of individual, bicrystalline grain boundaries, exhibit rather fast, liquid-like self-diffusion at high temperatures. Our simulations reveal that under relatively high tensile stresses these microstructures, indeed, exhibit steady-state diffusion creep that is homogeneous with a strain rate that agrees quantitatively with that given by the Coble-creep formula.
\end{abstract}

PACS numbers: 61.43.Dq, 61.72.Mm 


\section{Introduction}

Atomic structure of grain boundaries (GBs) was intensively studied in recent decades, particularly, in association with development of high-resolution microscopy techniques. Such experimental studies require formulation of structural models allowing for interpretation and understanding of the results. This need resulted in numerous atomic-level computational studies, which in combination with experiment provided detailed structural understanding of numerous grain boundaries in various materials $[1,2]$.

However, the majority of the high-resolution images were obtained for special grain boundaries, such as symmetric tilt GBs, where alignment of the atomic columns in the two crystalline grains adjacent to the GB plane enable to obtain information on GB structure. Furthermore, the experimental data on GB structure is typically available only for low (room) temperature structures. At high temperatures direct imaging of GB is still beyond experimental reach and only indirect information on GB structure is accessible, such as related to GB properties including diffusion, migration, or sliding.

By contrast to these experimental limitations atomic-level computer simulations are uniquely suited to study detailed atomic structure of virtually all kinds of GBs both at low and high temperatures. Furthermore, simulations can be performed in the complete absence of impurities and thus intrinsic properties can be separated from extrinsic properties to the much larger extent that is possible in experiment.

In the following we will present our general view of GB structure and related high-temperature behavior derived from our extensive simulation studies of numerous GBs and several model systems including silicon, fcc metal, and diamond. Finally we explore consequences of high-temperature GB diffusion on the interfacially controlled creep behavior nanocrystalline material.

\section{Low temperature GB structure}

Our atomic-level simulations of Si GBs revealed two distinct behaviors associated with low and high-energy grain boundaries [3]. The atomic structure of a representative high-energy GB obtained with the Stillinger-Weber (SW) empirical potential is shown in Fig. 1. Top panel in Fig. 1 corresponds to GB generated by a twist of perfect crystal structure around (100) direction with subsequent zero-temperature relaxation of atomic position. The structure appears to be rather distorted but one can still clearly identify atomic planes all the way to the GB plane. A more detailed analysis shows that the GB structure contains large amount of three-coordinated Si atoms corresponding to dangling bonds. Such configuration is highly unfavorable from the energetical point of view.

To obtain lower energy structures we explored two distinct procedures. In the first one we melted the GB structure over a large region and regrow the GB 

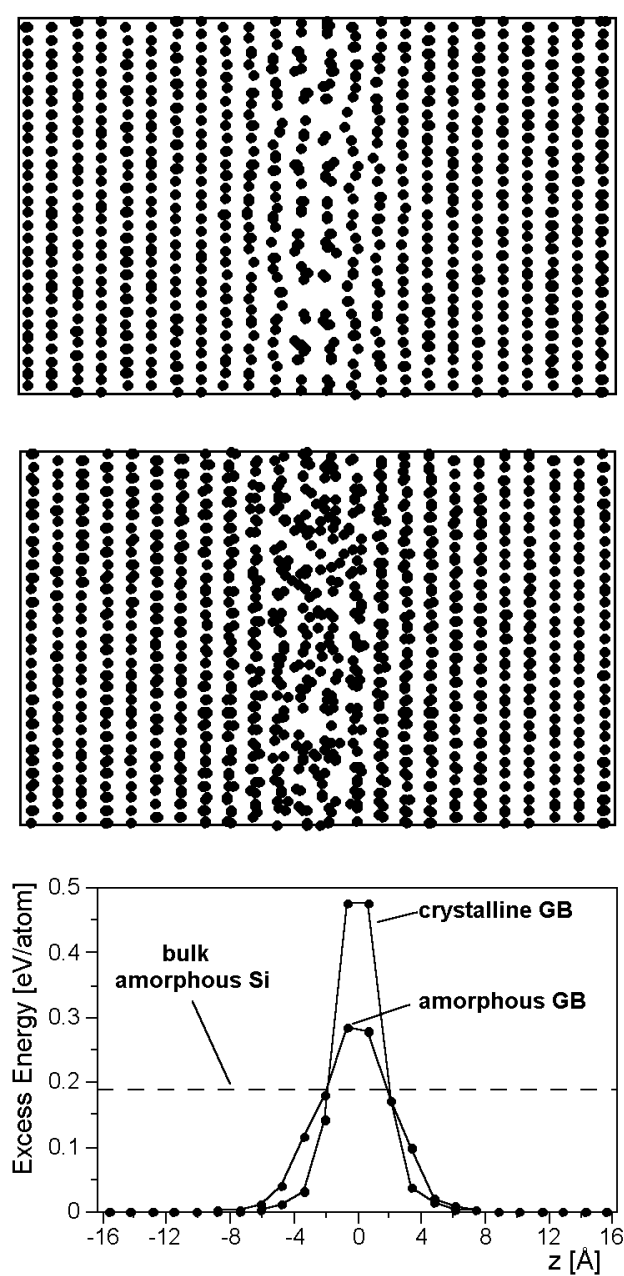

Fig. 1. Top panel: positions of the atoms projected onto the $x-z$ plane for the (100) $\phi=43.60^{\circ}(\Sigma 29)$ twist boundary in Si obtained by the zero-temperature relaxation. Middle panel: more disordered, yet lower energy high-temperature relaxed structures of the same GB. Bottom panel: energy profiles for zero and high temperature relaxed GBs along with the corresponding energy of bulk amorphous Si demonstrate the origin of the driving force for the GB disordering.

from the melt. Then we cooled the structure down to zero temperature. In the second approach we heated the GB structure to about $85 \%$ of the melting point and then cooled down to zero temperature. In all cases these two high-temperature treatments yielded GB structures with practically the same overall characteristics although the detailed atomic structures and GB energies were not completely identical. 
The high-temperature relaxed structures (Fig. 1, middle panel) exhibit much more structural disorder than the zero- $T$ relaxed structure (Fig. 1, top panel). However, the energy of the high- $T$ relaxed structures is about $15 \%$ lower than zero- $T$ relaxed structures. This lowering of the GB energy despite of the introduction of a large degree of spatial disorder is accompanied by the elimination of dangling bonds and thus recovering fourfold coordination of Si atoms. Remarkably local atomic structure in the disordered GB core is very similar to the local structure of bulk amorphous silicon [3].

Further insight into the origin of GB disordering is provided in the lower panel of Fig. 1 showing profiles of the plane-by-plane average energy per atom. The energy profile of the zero- $T$ relaxed structure is rather narrow and it has large maximum value. The profile of the high- $T$ relaxed GB is broader but with much lower peak value. Also indicated in the figure by the dashed line is the cohesive energy of bulk amorphous silicon prepared by slow cooling of the liquid phase. This provides the indication of the driving force for the GB disordering. The high peak energy of zero- $T$ relaxed structure by far exceeds the average energy for bulk amorphous material. By contrast, in the most disordered plane of the high- $T$ relaxed structure the peak in the energy profile is much closer to the cohesive energy of amorphous Si. More importantly, the GB energy (i.e., the integral under the peak) is lowered during disordering despite a broadening of the GB.

Based on these insights, one might expect that all high-energy GBs should have similar, "confined-amorphous" structures. To test this prediction we investigated three other high-angle, high-energy twist boundaries with qualitatively different zero-temperature relaxed structures. We found that high-temperature relaxation lowers the energies of all these GBs. Moreover, despite being sandwiched between different crystallographic surfaces, all GBs were found to be disordered and very similar to each other, as is also apparent from the related energy profiles in Fig. 2.

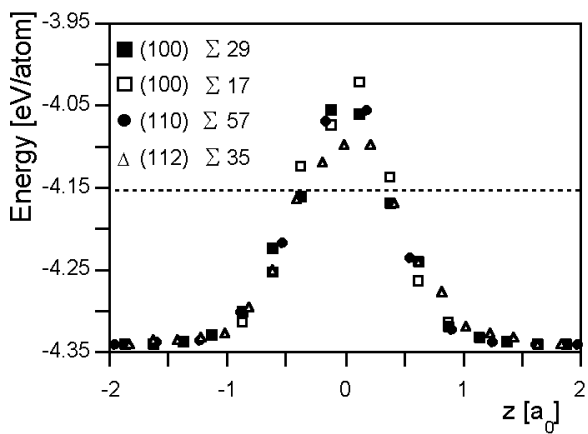

Fig. 2. Energy profiles along the $z$ direction for four different high-energy, large-angle GBs in Si demonstrating universal nature of high-energy GBs. 
To further confirm our predictions we also performed studies of the same high-energy GBs with Tersoff's empirical Si potential [4] as well as with quantum-mechanics based empirical tight-binding simulations [5]. In all cases we found that whereas details of the atomic structures and energetics vary from one system description to the other, in general the results and conclusions are the same as those obtained with the SW potential.

By contrast with these disordered structures of high-energy GBs, one might expect that a low-energy GB should not lower its energy upon high-temperature equilibration and hence should not disorder. To confirm this prediction we studied the representative low-energy GBs which are also twist GBs, however on the (111) plane which is the most widely-spaced (and hence, lowest-energy [3]) plane in the diamond structure. Indeed, as expected, neither its structure nor its energy change during high-temperature annealing and the GB remains crystalline right up to the GB plane.

We also performed extensive simulations of fcc metal GB structures [6]. Quite remarkably the results are analogous to those obtained for silicon with high-energy GBs exhibiting large degree of structural disorder which is very similar to that present in metallic glasses. Also as in the case of Si, low energy grain boundaries are crystalline and ordered [6].

By contrast to $\mathrm{Si}$ and fcc metals situation is somewhat different in carbon diamond GBs. Using Monte Carlo simulations and Tersoff's carbon potential we demonstrated that the greater bond stiffness in diamond combined with its ability to change hybridization in a defected environment from $s p^{3}$ to $s p^{2}$ lead to different GB structures as in purely $s p^{3}$-bonded silicon [4]. We found that in high-energy diamond GB even relatively small bond distortions exact a considerable price in energy that favors a change to $s p^{2}$-type local bonding; these competing effects translate into considerably more ordered diamond GBs than Si GBs. Structural disorder in the Si GBs is therefore partially replaced by coordination disorder in the diamond GBs.

Our follow up studies of diamond GBs by means of tight-binding molecular dynamics (MD) simulations confirmed existence of large $s p^{2}$ component in their atomic structure [7]. We also demonstrated that this $s p^{2}$-bonding gives rise to localized bands within the energy band gap, which via multiphonon assisted hopping conduction may lead to the experimentally observed conductivity and electron emission in nanocrystalline diamond.

\section{High-temperature GB structure and properties}

The presence of disordered GB films in thermodynamic equilibrium suggests that upon heating above the glass-transition temperature, $T_{\mathrm{g}}$, the thin amorphous intergranular phase can undergo an amorphous-to-liquid transition analogous to the inverse glass transition. Indeed, we observed in MD simulations that above $T_{\mathrm{g}}$, 
the confined amorphous phase undergoes a reversible structural and dynamical transition to a confined liquid [8]. By contrast with the bulk glass transition, this thermodynamic-equilibrium transition is continuous and thermally activated, starting at $T_{\mathrm{g}}$ and complete at the melting point, $T_{\mathrm{m}}$, at which the entire boundary is liquid [8].

The amorphous-to-liquid transition of the confined GB phase results in the very fast GB diffusion that is characteristic of the liquid rather than the solid. An Arrhenius plot shown in Fig. 3 for grain boundary diffusion for high-energy bicrystalline GBs in silicon yields relatively low activation energy of $1.4 \pm 0.1 \mathrm{eV}$. However, as also shown in Fig. 3, the activation energy for diffusion in the Si melt is still lower and equal to about $0.7 \mathrm{eV}$. This apparent discrepancy originates from the fact that the amount of fast-diffusing liquid increases with temperature. In Fig. 4 we present excess GB volume as a function of temperature for high-energy GB showing decreasing GB volume with increasing temperature. This decreased volume is a signature of formation of an Si liquid, which has higher coordination and density than that of either crystalline or amorphous silicon.

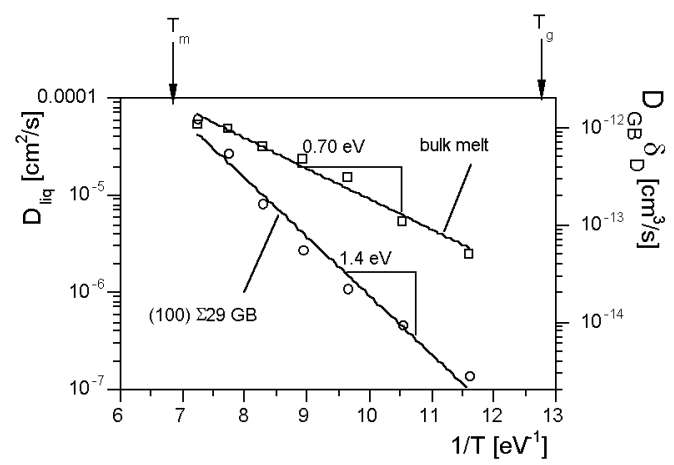

Fig. 3. Arrhenius plot for self diffusion in the (100) $\Sigma 29$ GB (right scale) and in the bulk, supercooled melt (left scale). Right scale: $D_{\mathrm{GB}} \delta_{\mathrm{D}}$ is the diffusion flux in the GB with a "diffusional width" $\delta_{\mathrm{D}}$.

The above observations suggest that the activation energy of $\approx 1.4 \pm 0.1 \mathrm{eV}$ for GB diffusion in high-energy Si GB above $T_{\mathrm{g}}$ involves two distinct processes. The first is thermally activated formation of the confined liquid from the confined-amorphous phase. Indeed, from the volume contraction we calculated the activation energy for the formation of the liquid to be $0.65 \mathrm{eV}$. The second is the subsequent bulk-liquid-like atom migration, with activation energy of $0.70 \mathrm{eV}$. We therefore conclude that above $T_{\mathrm{g}}$, the mechanism for self-diffusion in the high-energy GB is rather similar to that in the bulk melt. Consequently, the activation energy for GB diffusion is equal to the activation energy for the formation of the liquid and the activation energy for the migration in the liquid [8]. 


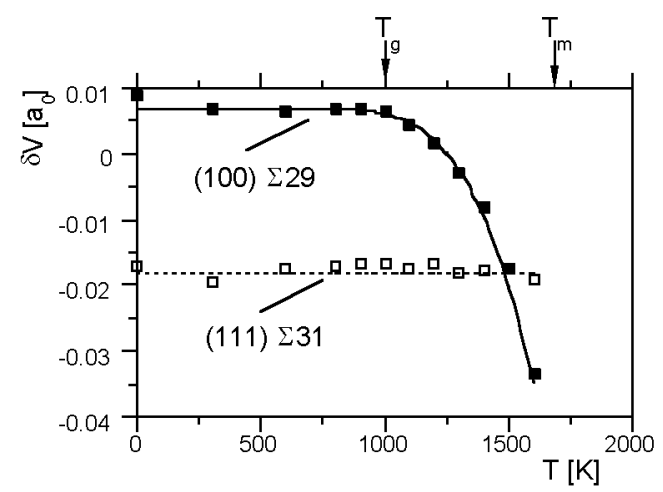

Fig. 4. Temperature dependence of the volume expansion per unit GB area, $\delta V$ (in units of the zero-temperature lattice parameter) for the high-energy (100) $\Sigma 29$ and low-energy (111) $\Sigma 31$ twist GBs in silicon. The bulk glass-transition temperature, $T_{\mathrm{g}}$, and melting point, $T_{\mathrm{m}}$, are indicated on the top axis.

Also shown in Fig. 4 is excess GB volume as a function of temperature for a low-energy Si GB. In this case there is no signature of any transformation and this low-energy GB remains crystalline all the way up to the melting point.

Our studies of GB diffusion in fcc metals demonstrated that they behave analogously to silicon GBs [6]. Remarkably, at high temperatures the few representative high-energy, high-angle (tilt or twist) boundaries examined by us exhibit the same, rather low self-diffusion activation energy and an isotropic, liquid-like diffusion mechanism that is independent of the boundary misorientation [6]. Again as in the case of silicon low-energy GBs remain crystalline all the way up to the melting point. Very interesting results were obtained for GBs with high but not the highest possible energies. In this case the GB structure is crystalline at low tem-

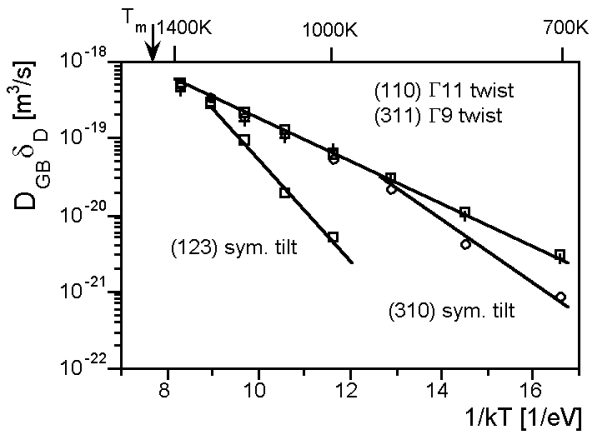

Fig. 5. Arrhenius plots for the diffusion flux, $D_{\mathrm{GB}} \delta_{\mathrm{D}}$, between $700 \mathrm{~K}$ and $1400 \mathrm{~K}$ for four Pd GBs. The melting point for the Pd (embedded atom method, EAM) potential is estimated to be about $T_{\mathrm{m}} \approx 1500 \mathrm{~K}$. The two highest energy GB exhibit fast diffusion and low activation energy. Lower energy (but still relatively high) GBs shows change in the slope indicating structural GB transition. 
peratures but undergoes a disordering structural transition at high temperature. Consequently these GBs exhibit anisotropic and GB dependent diffusion with high activation energies at low temperatures. At high temperatures they all exhibit the same isotropic liquid-like diffusion with a universal low activation energy (see also Fig. 5).

\section{GB diffusion creep}

Application of a constant, e.g. tensile stress, $\sigma$, to a polycrystalline material with an average grain size, $d$, at high-temperature, $T$, usually results in steady-state creep; i.e., a time-dependent plastic deformation characterized by a constant strain rate, $\varepsilon(T, \sigma, d)$. Depending on the magnitudes of $T, \sigma$, and $d$, the underlying mechanism for steady-state creep may involve the diffusion of atoms through the lattice or along the grain boundaries, the thermally activated glide or climb of dislocations, or interfacial processes, such as grain sliding [9].

Here we limit ourselves to the investigation of diffusion creep in model nanocrystalline microstructures. As is well known, the steady-state rate of diffusion creep may either be limited by lattice diffusion through the grain interiors or by interfacial diffusion along the GBs, characterized by the diffusion constants $D_{\mathrm{L}}$ and $D_{\mathrm{GB}}$, respectively. The net result is the same for both mechanisms, namely a homogeneous deformation resulting in the elongation of the grains. The creep rate due to lattice diffusion through the grain interiors (Nabarro-Herring creep) is given by [9]:

$$
\dot{\varepsilon}=10 \frac{\sigma \Omega_{\mathrm{D}}}{k T} \frac{D_{\mathrm{L}}}{d^{2}} .
$$

By contrast, the creep rate due to GB diffusion (Coble creep) is given by [9]:

$$
\dot{\varepsilon}=47 \frac{\sigma \Omega_{\mathrm{D}}}{k T} \frac{\delta_{\mathrm{D}} D_{\mathrm{GB}}}{d^{3}} .
$$

Here $\delta_{\mathrm{D}}$ is the effective diffusional width of the GB. $\sigma \Omega_{\mathrm{D}}$ is the work performed by the stress during an elementary diffusion jump in the lattice or GB, respectively. The activation volume, $\Omega_{\mathrm{D}}$, therefore depends on the diffusion mechanism through the lattice or along the GBs, and is usually thought to be of the order of the perfect-crystal atomic volume, $\Omega$. (For the cubic diamond lattice, $\Omega=a_{0}^{3} / 8$, where $a_{0}$ is the zero-temperature lattice parameter.)

Because of its stronger increase with decreasing grain size, for the smallest grain sizes Coble creep usually dominates over Nabarro-Herring creep. For example, according to Eq. (2) the strain rate for Coble creep increases by six orders of magnitude as the grain size is reduced, say, from $1 \mu \mathrm{m}$ to $10 \mathrm{~nm}$ whereas, at the same temperature, the strain rate for Nabarro-Herring creep increases by only four orders of magnitude. Based on this strong increase in $\varepsilon$, with decreasing grain size, one might expect that for a grain size of nanometer dimensions the steady-state strain rate is high enough to be observable by molecular-dynamics simulation, thus enabling one to test the physical assumptions underlying the Coble formula (2). 
Due to the limitation of MD simulations to a time scale of typically $10^{-9} \mathrm{~s}$, the detection of a total strain of $1 \%$ in such simulations would require a steady-state creep rate of $10^{7} \mathrm{~s}^{-1}$. Based on Coble's formula, such extremely high creep rates, indeed, seem achievable in MD simulations, provided the simulated microstructures are stable with respect to grain growth and are under the relatively high stresses.

As described in Sec. 3, high-energy GBs exhibit very fast liquid-like GB diffusion at high temperature. Therefore we tailored our input silicon nanostructures to have a uniform grain size and grain shape and to contain only high-energy GBs. Our model nanocrystalline input microstructures consist of only four grains of identical size and shape arranged in a three-dimensionally periodic simulation cell. These microstructures were grown from a melt into which small, randomly oriented crystalline seeds were inserted and subsequently cooled below the melting point [10]. So as to avoid grain growth during the subsequent creep simulation, the four seeds were placed near the face-centered-cubic sites of an initially cubic simulation cell; this arrangement results in a final microstructure that has a uniform dodecahedral (i.e., close to spherical) grain shape and grain size (of 3.8, 5.4, and $7.3 \mathrm{~nm}$ ).

Analysis of the GB structure in such synthesized models of nanocrystalline material revealed the presence of highly disordered intergranular films of constant, uniform thickness, $\delta$, of about $0.5 \mathrm{~nm}[10]$ which is essentially the same as that in high-energy bicrystalline GBs. This allows us to estimate the Coble creep rate in our idealized microstructures. Let us assume $T=1200 \mathrm{~K}$ (at which our bicrystal simulations give a value of $\delta_{\mathrm{D}} D_{\mathrm{GB}} \approx 2 \times 10^{-20} \mathrm{~m}^{3} / \mathrm{s}$ [8]), a grain size of $d=5 \mathrm{~nm}$, and a relatively high tensile stress of $\sigma=1.00 \mathrm{GPa}$ (i.e., about two orders of magnitude higher than typical experimental stresses - but still well below the theoretical strength of $\mathrm{Si}$ of about $30 \mathrm{GPa}$ ). Inserting these values into Eq. (2) yields a strain rate for Coble creep of about $\varepsilon \approx 1 \times 10^{7} \mathrm{~s}^{-1}$, which lies indeed within a time frame accessible to MD simulation. At first sight such a high strain rate may seem a bit unrealistic or even nonphysical. However, by simply decreasing the applied stress by two orders of magnitude and increasing the grain size, say, to $1 \mu \mathrm{m}$ while assuming that the underlying physics remains the same, one obtains more realistic values of $\varepsilon \approx 10^{-2} \mathrm{~s}^{-1}$.

In our deformation simulation we applied a constant-stress algorithm that allows for elongation of the rectangular simulation cell in the tensile $(z)$ direction and Poisson contraction perpendicular to it (in the $x-y$ plane). A typical plot of the diagonal strains, $\varepsilon_{x x}, \varepsilon_{y y}$, and $\varepsilon_{z z}$ vs. simulation time, $t$, is shown in Fig. 6 for the system with the intermediate grain size at $T=1400 \mathrm{~K}$ and $\sigma=1 \mathrm{eV} / a_{0}^{3}$. According to the figure, following rapid elastic straining both the tensile and Poisson strains vary linearly with simulation time, indicating that steady-state creep behavior is, indeed, reached and that a strain rate can reliably be extracted from the simulations [11]. 


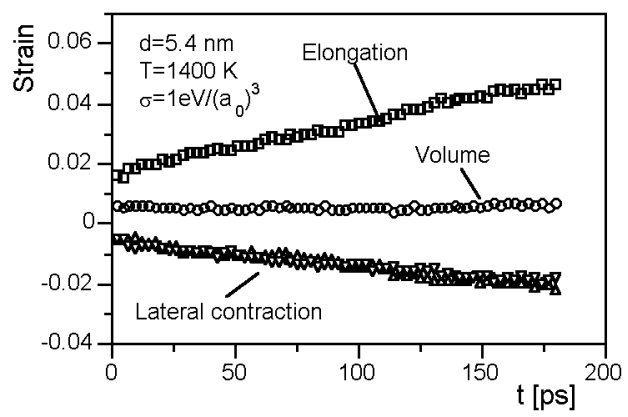

Fig. 6. Typical plot of the total (elastic + plastic) strain and associated system volume vs. simulation time for the system with the intermediate grain size at $T=1400 \mathrm{~K}$ and $\sigma=1 \mathrm{eV} / a_{0}^{3}$. The slopes of the linear $\varepsilon(t)$ curves yield the strain rate, $\dot{\varepsilon}$. The elastic strains, $\varepsilon(t \rightarrow 0)$, are governed by the underlying Young modulus and Poisson ratio.
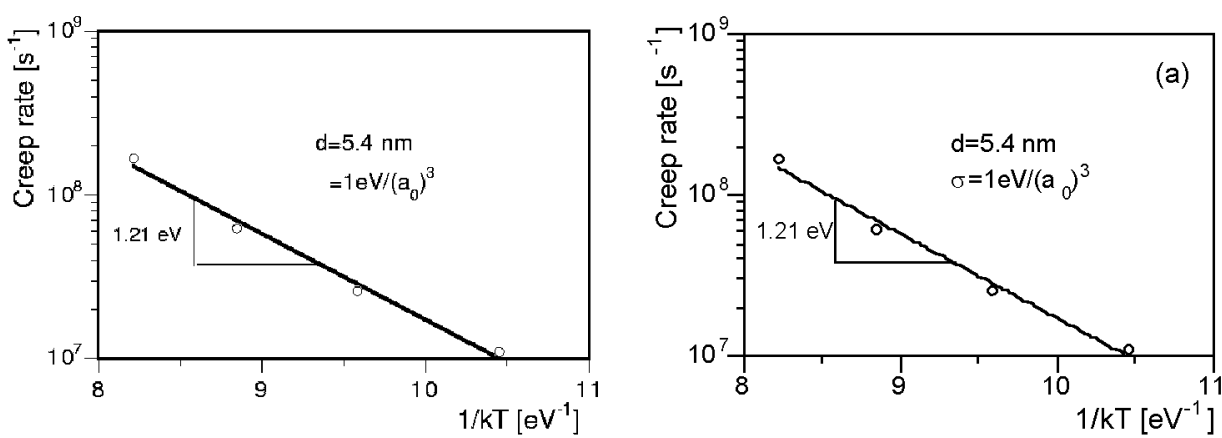

Fig. 7. Arrhenius plot for the creep rate between 1100 and $1400 \mathrm{~K}$ for our model $\mathrm{Si}$ microstructure with the intermediate grain size. (b) Arrhenius plot for the GB diffusion flux, $D_{\mathrm{GB}} \delta_{\mathrm{D}}$, for the same system as in (a), however, under zero external stress.

Insight into the nature of the underlying creep mechanism can be gained from the study of the temperature dependence of the creep rate. As seen from the Arrhenius plot in Fig. 7, the creep rate is thermally activated, with an activation energy of $1.21 \pm 0.1 \mathrm{eV}$. According to Eq. (2), this activation energy should be the same as that for GB diffusion. As discussed before, an Arrhenius plot for $D_{\mathrm{GB}} \delta_{\mathrm{D}}$ for high-energy bicrystalline GBs in silicon yields an activation energy of $1.4 \pm 0.1 \mathrm{eV}$ [8], which differs slightly from the above creep value for our nanocrystalline microstructures (although, within the error bars, the two values are practically the same). To investigate the origin of this slight discrepancy, we performed independent simulations of self-diffusion in our model microstructures in the absence of an applied stress and obtained an activation energy of $1.19 \pm 0.1 \mathrm{eV}$, in excellent agreement with the activation energy of $1.2 \pm 0.1 \mathrm{eV}$ for GB creep [11]. The small difference between the activation energies for $D_{\mathrm{GB}} \delta_{\mathrm{D}}$ in the bicrystalline $\mathrm{GB}$, on the one hand, and the GBs in the nanocrystalline microstructure, on the other, 
might be indicative of a role played by the grain junctions in the limit of very small grain sizes. However, in view of the fundamental differences between the two systems as far as microstructural constraints are concerned, the reasonably good agreement of both the activation energies and absolute values for $D_{\mathrm{GB}} \delta_{\mathrm{D}}$ determined by MD simulation is rather encouraging.

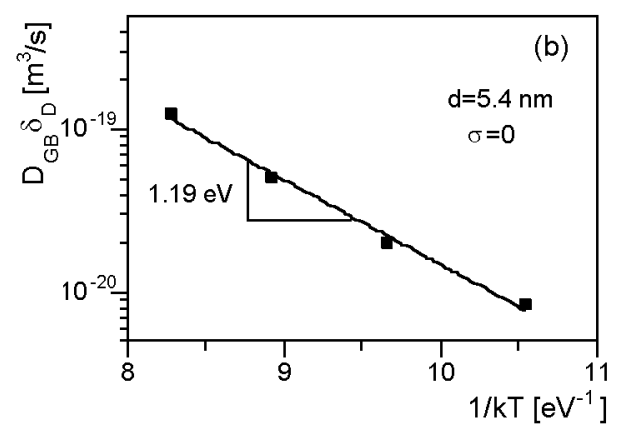

Fig. 8. Log- $\log$ plot of the creep rate vs. grain size. For the two largest grain sizes studied, the creep rate approaches the $1 / d^{3}$ dependence predicted by the Coble formula (2).

Another critical element of the Coble-creep formula (2) is the $d^{-3}$ variation of the creep rate. According to Fig. 8, for the two largest grain sizes (of $d=5.4$ and $7.3 \mathrm{~nm}$ ) the creep rate, indeed, exhibits this $d^{-3}$ dependence. By contrast, the creep rate determined for the smallest grain size (of $d=3.8 \mathrm{~nm}$ ) clearly deviates from this behavior. This deviation is likely due to the fact that for this small grain size, the disordered GB phase accounts for over $40 \%$ of the overall system volume, whereas the Coble formula was derived under the assumption of the GB width being much smaller than the grain size.

\section{Summary}

In this paper we discussed our extensive atomistic studies of grain boundaries in silicon, fcc-metal and carbon diamond. Based on these studies we concluded that in general high-energy GBs tend to be disordered at low temperatures and their local atomic structure is similar to that of corresponding bulk amorphous materials. At high temperatures these disordered structures undergo a transition to confined liquid structures and are characterized by a very fast diffusion. Intermediate energy GBs may be crystalline at low temperatures and become liquid-like at high temperatures. Finally low energy GBs are crystalline all the way up to the melting point.

We were also able to design models of nanocrystalline material which in the limit of large stress, high temperature and a small grain size, allow for direct observation of a steady-state GB diffusion creep. We showed that the creep behavior 
is controlled by GB diffusion and described quantitatively by the Coble creep formula. In particular, in accordance with the Coble formula, the activation energy for GB diffusion creep is the same as that for GB self-diffusion, and the strain rate increases with decreasing grain size as $1 / d^{3}$. However, with presently available computational resources, typical total creep strains in our simulations were only of the order of $1-2 \%$. We can therefore not exclude the possibility that both the microstructure and the atomic structures of the GBs may change significantly for larger total strains and longer deformation times. Given the ever so rapidly increasing computational speeds and available computer memories, it appears that in future investigations the constraints imposed on our designed model microstructures can gradually be removed. This might enable a step-by-step approach towards elucidation, at the atomic level, of the interplay between GB-sliding and GB-diffusion controlled creep deformation, on one hand, and stress and/or temperature-induced microstructural evolution, on the other. It seems to be the complex interplay of such essentially GB diffusion controlled processes that is thought to be responsible for the still not very well understood superplastic behavior of fine-grained metals and ceramics.

\section{References}

[1] A.P. Sutton, R.W. Balluffi, Interfaces in Crystalline Materials, Clarendon Press, Oxford 1995 .

[2] Materials Interfaces: Atomic-level Structure and Properties, Eds. D. Wolf, S. Yip, Chapman and Hall, London 1992.

[3] P. Keblinski, S. R. Phillpot, D. Wolf, H. Gleiter, Phys. Rev. Lett. 77, 2965 (1996); J. Am. Ceram. Soc. 3, 717 (1997).

[4] P. Keblinski, S.R. Phillpot, D. Wolf, H. Gleiter, J. Mater. Res. 13, 2077 (1998).

[5] F. Cleri, P. Keblinski, L. Colombo, S.R. Phillpot, D. Wolf, Phys. Rev. B 57, 6247 (1998).

[6] P. Keblinski, D. Wolf, S.R. Phillpot, H. Gleiter, Scr. Metall. 41, 631 (1999).

[7] F. Cleri, P. Keblinski, L. Colombo, S.R. Phillpot, D. Wolf, Europhys. Lett. 46, 671 (1999).

[8] P. Keblinski, D. Wolf, S.R. Phillpot, H. Gleiter, Philos. Mag. Lett. 76, 143 (1997).

[9] See e.g., F. R. Nabarro, H.L. de Villiers, The Physics of Creep, Taylor and Francis, London 1995.

[10] P. Keblinski, S.R. Phillpot, D. Wolf, H. Gleiter, Acta Mater. 45, 987 (1996).

[11] P. Keblinski, D. Wolf, H. Gleiter, Interface Sci. 6, 205 (1998). 